\title{
Situated Learning: A Theory for Learning Situation Awareness
}

\author{
Donna Forsyth Wilt and Philip Horton \\ Florida Institute of Technology
}

\begin{abstract}
In aviation, situation awareness is the accurate perception of the factors and conditions affecting the aircraft and flight crew. The pilot's situation awareness in the flight environment is recognized as an important factor in flight safety, yet no known training method exists for improving situation awareness in novice pilots. The educational theory of situated learning is based on the idea that learning takes place as an interaction between a novice, an expert, and their social and physical environment. This theory has many commonalities to flight training with the student as the novice, the instructor as the expert, and the flight training environment as the physical environment. Based on this theory, the theories of situated learning are related to the flight environment and the way in which these educational theories could be used as a foundation in developing instructional techniques to improve situation awareness.
\end{abstract}

\section{Situation Awareness and Its Importance}

Situation Awareness (SA) is a cognitive process that occurs in many situations. SA has various definitions, but in aviation, most definitions share the common theme that SA is the accurate perception of the factors and conditions affecting the aircraft and flight crew (Edens, 1991). An operational definition of SA is when the pilots know (a) the state of their own aircraft, (b) the person or thing in charge-the pilot or an automated system, (c) the evolution of events over time, (d) the spatial relationships among aircraft and other objects, and (e) the presence of threat and their objectives (Harwood, Barnett \& Wickens, cited in Fracker, 1988). Endsley (1988) describes three levels of SA: Level 1 is the perception of some element in the environment, Level 2 is the elements being put together to form patterns and a holistic picture of the environment, and Level 3, the highest level, is the projection of the elements of the environment into the near future.

In some instances, SA can be a matter of life or death. Accidents caused by pilot error far outnumber accidents caused by mechanical problems in aviation today. As technological advances have made aircraft more reliable, the percentage of accidents due to malfunctions has decreased, while the percentage of accidents due to pilot error has increased (Forsyth and Shaughnessy, 1978).

When a pilot does not have an accurate perception of the factors and conditions affecting the aircraft and flight crew, performance can suffer, mistakes can be made, and accidents can result. The risk of poor performance increases with poor SA (Endsley, 1993). Therefore the aviation community strongly believes that increasing a pilot's SA will improve pilot performance and improve safety (Schwartz, 1987). 
Two areas for research in increasing a pilot's SA are cockpit design and education. Current studies related to SA have been from a psychological and human factors point of view and have focused on finding better definitions for SA and measuring SA (Crabtree, Marcelo \& McCoy, 1993; Endsley, 1988, 1990a, 1990b; Fracker, 1988; Hartman \& Secrist, 1991). Now that the meaning and measurement of SA has been clarified, SA can be studied and researched from an educational perspective.

The models of SA agree that SA occurs when patterns perceived in the environment are matched to schema in long-term memory (Fracker, 1988; Kass, Herschler I Companion, 1990; Endsley, 1988). The model proposed by Stokes, Kemper, and Marsh (1992) adds the idea that not only must schema be matched, but the pilot must have the ability to determine which cues in the environment are relevant to perceiving patterns. Based on the model developed by Stokes and his colleagues, inexperienced pilots lack two things: the repertoire of schemata, and the ability to determine which cues are relevant.

When Stokes, Kemper, and Marsh (1992) tested novice and expert pilots, they found two interesting correlations. First, they found no difference in the inherent cognitive abilities of novice versus expert pilots. This implies that SA is not an inherent skill. Second, they found that certificates (private, private with instrument rating, commercial pilot, flight instructor, or air transport pilot) were a better predictor of relevant cue recognition than total flight hours (for certificates, $s r^{2}=0.49, n=26, p<0.001$ ).

One method to improve SA is to design new systems and displays. However, as noted by Hartman and Secrist (1991), these systems and displays tend to have little impact on the larger aviation community and tend be aircraft specific. An alternative approach for improving SA is to treat SA as a generic skill exercised by all pilots. The findings by Stokes et al., which state that SA is not an inherent skill, are consistent with the idea that $\mathrm{SA}$ is a teachable skill. If SA is a generic skill, then pilots can then be trained for enhanced SA that will be aircraft independent (Hartman \& Secrist, 1991).

Typical flight training programs (private certificate or instrument rating) do not specifically address SA at all. The flight portion of the training is designed to teach the mechanical and procedural skills of flying. The ground portion of the training, at best, only raises awareness of SA with respect to the decision-making process (Instrument Commercial Manual, 1994). The Instrument Commercial Manual (1994), a popular textbook for instrument and commercial ground school, points out the importance of SA. Although the manual states that good decision making is predicated on having good SA, it gives no advice on how to achieve it. To achieve SA, the theory of situated learning provides a foundation around which SA can be learned when added to flight training curriculums. 


\section{Situated Learning}

\section{The Indexicality of Knowledge}

Situated learning is built around the concept of the indexicality of knowledge. In indexicality, pieces of knowledge each refer to, point to, or index, some part of the world; and these pieces are inextricably a product of the activity and situations in which they are produced (Brown, Collins \& Duguid, 1989). This concept implies that knowledge is inherently embedded in the situation. Therefore, learning methods must be similarly embedded in realistic situations. Brown and his colleagues draw an analogy between knowledge and a jig-saw puzzle. Knowledge is coded by and connected to the activity and environment, just as the pieces of the puzzle are coded by the picture and the shape of the pieces. Using this analogy, SA is accurately putting together the jigsaw puzzle of knowledge.

\section{Authentic Activity in Flight Training}

In situated learning, activities that preserve and present the jigsaw puzzle of knowledge and are coherent, meaningful, and purposeful are given the name "authentic activities" (Brown et al., 1989). In aviation, authentic activities are those activities involved in a flight from one airport to another and the realistic activities that should or might occur on the flight. According to Brown et al., when activities are transferred to the classroom setting, typically they are distorted and become part of the school culture instead of the authentic culture. When taking a task from real life to the classroom, an attempt is often made to separate the salient features from the peripheral "noise." But according to Brown et al., the context of the activity is extraordinarily complex. Essential support is drawn from the complex setting, and it is impossible to know what "noise" can be separated out. So the classroom activity is not only missing some of its important features, the students may also come to rely on features that appear in the classroom context that would not appear in the authentic activity.

In situated learning, the emphasis is on learning not teaching. Lave and Wenger (1991) go so far as to define a "learning curriculum" as one that is comprised of situated opportunities for the improvisational development of a new practice or goal. "The practice of the community creates the potential 'curriculum' in the broadest sense. ... A learning curriculum unfolds in opportunities for engagement in practice" (p. 93). A learning curriculum is a set of learning resources, not something that can be considered in isolation, manipulated in arbitrary didactic terms, or analyzed apart from the context of its environment.

Knowledge of the situation in the flight environment agrees well with the concepts of the indexicality of knowledge and authentic activity. However, contemporary flight lessons are a juxtaposition of maneuvers and procedures that would never occur together on a real trip (Aviation Instructor's Handbook, 1977; Kershner, 1989). Missing from the lessons are 
the coherent navigation, orientation, and communication activities in the context of an authentic trip. Much of the indexical knowledge that is embedded in a real trip and is used as part of SA has been omitted or subordinated in the local flight lesson. The typical situation that exists in a lesson to practice a flight maneuver or procedure is different from the situation when the maneuver or procedure is actually needed or encountered in a real flight. The salient features used to determine SA cannot be separated from the noise in the environment in which it occurs. The same piece of knowledge that is crucial in one situation may be unimportant in another situation. This distortion of authentic activities applies even when the classroom is an airplane or simulator. The implication is that SA must be experienced in the context of real events in which consequences can unfold and schemata can be built. Schemata that are used to determine SA must be as authentic as possible. As stressed by Brown et al., the learning methods must also be embedded in authentic situations.

Within situated learning, the general methods to support learning are based on Vygotsky's cognitive theory of "zones of proximal development" (Lave \& Wenger, 1991), on the expert-novice relationship between teacher and student (Brown et al., 1989, 1993; Greenfield, 1984, Lave \& Wenger, 1991; Rogoff \& Gardner, 1984), and on a teaching technique called "scaffolding" (Greenfield, 1984). Parallels can easily be drawn between the methods proposed by situated learning and the flight training of novice pilots.

\section{Zone of Proximal Development}

Vygotsky's "zone of proximal development" is the distance between the learner's ability when working independently and the learner's ability when assisted by or collaborating with a more experienced person. The zone of proximal development defines the formative stages of the knowledge that exists for the learner. According to Vygotsky, the learner first carries out an activity in cooperation with a teacher; that activity then has to be mastered inter-individually before it can be mastered intra-individually. The learner, in working with the experienced person, not only solves a problem that could not be solved alone, but also moves closer to being able to do it alone (Greenfield, 1984).

In flight training, zone of proximal development is apparent in early lessons in a curriculum when the instructor provides substantial coaching to enable the student to complete a task. As the student progresses, the instructor assists the student less and less. Zone of proximal development is also apparent in flight lessons when a situation arises beyond the scope of the lessons, and the instructor "talks the student through it" rather than the instructor doing the activity for the student. Such a situation is an important learning opportunity because the student performs a task that couldn't be done alone, and the student also moves closer to being able to do the task alone. In such situations, the instructor, acting as an expert, helps the novice student, much the way an expert mentors an apprentice. 


\section{Elight Instructor as Expert Pilot}

As illustrated above, the student-flight instructor relationship is an apprenticeship-type relationship even though it is not usually thought of in these terms. In the classroom setting, the flight instructor is the expert, regardless of the instructor's experience, while the student is the novice, regardless of the student's prior ratings. The descriptions of apprenticeship-type learning in the literature agree with the way much of the learning takes place in the cockpit.

Lave and Wenger (1991) emphasize that in apprenticeship the novice is given peripheral tasks that can be done with the novice's skill level, while the overall task is beyond the skill level of the novice. At the same time, the novice observes the expert, works with the expert, and gradually picks up the expert's knowledge. This learning process is referred to by Lave and Wenger as "legitimate peripheral participation."

A concept that is similar to legitimate peripheral participation but captures the student doing more than just peripheral tasks (such as SA in flight training) is "proleptic instruction." Proleptic instruction, defined by Wertsch and Stone (as cited in Rogoff \& Gardner, 1984), takes place when a novice learns information and skills by observing an expert while participating at a comfortable, but slightly challenging, level. Proleptic teaching is an integration of explanation and demonstration with an emphasis on the learner's participation in the activity. Proleptic instruction is a deliberate but tacit process that the participants construct in the course of communication. As with legitimate peripheral participation, when the novice performs a task under the expert's guidance, the novice participates in creating his or her own contextual knowledge while at the same time acquiring some of the expert's understanding of the situation (Rogoff \& Gardner, 1984). This could also be thought of as the novice "stealing" the important knowledge from the expert, the way an aspiring football player steals a move from the star player (Brown \& Duguid, 1993).

Legitimate peripheral participation and proleptic instruction both include the transfer of responsibility for the management of the task from the expert to the novice as a crucial feature of the learning process (Rogoff $\&$ Gardner, 1984). To turn over responsibility, the expert must be sensitive to changes in the novice's zone of proximal development and provide only enough support to bridge the gap between what can be done by the novice alone and what can be done with the expert's help.

In flight training, the instructor, as expert, can provide guidance, serve as an example to the student, and pass along important knowledge. Handling radio communications is an example of proleptic instruction. The instructor has the student handle routine communications and assists as needed to handle the more complicated transmissions. Through the course of conversation, the instructor passes on his or her own knowledge of techniques and procedures for communicating and managing the radios. As the student 
progresses, the instructor reduces the support and increasingly turns over responsibility for radio communications to the student.

\section{Elight Instructor as Scaffolding}

Radio communications is not the only area in which the instructor provides support. In general, the flight instructor provides support in many ways for the flight student to enable a successful flight lesson, and the instructor always has overall responsibility for the flight. With SA in particular, the instructor, as the expert, initially takes on the responsibility of SA while emphasizing the learning of procedures and psychomotor skills. The instructor provides support for SA by filling in the student's "mental picture" as needed. As the student progresses, the responsibility for maintaining SA is progressively turned over to the student.

An analogy can be drawn between the role of the instructor in providing support and the scaffolding used in construction. The metaphor of an instructor as scaffolding was originated by Wood, Bruner, and Ross in 1976 (as cited in Greenfield, 1984), and a whole theoretical model for teaching has evolved around it. Greenfield (1984) emphasizes five things teachers have in common with construction scaffolding: (a) both provide support, (b) both function as a tool, (c) both extend the range of the worker, (d) both allow the worker to accomplish a task not otherwise possible, and (e) both are used selectively to aid the worker where needed. She also points out, however, that this analogy breaks down when considering that a teacher helps the student learn and thereby eliminates the need for scaffolding. A physical scaffolding could never eliminate the need for its own existence.

The key to success with scaffolding is two-fold. First, the instructor must determine just how much support the student needs and provide this appropriate level of support. Then, as the student's capabilities increase, the instructor must decrease the support to match the student's decreasing needs and let the student assume responsibility. The instructor's support should be calculated so that the student is always at a level just beyond that which the novice could manage independently (Rogoff \& Gardner, 1984).

Scaffolding is closely related to the idea of cooperative learning between an experienced and inexperienced person. Scaffolding represents an imbalance of responsibility in which the inexperienced persons have as much responsibility as they can handle. The experienced person has a greater responsibility for the successful accomplishment of the task and compensates for the inexperienced person's weaknesses (Greenfield, 1984).

Scaffolding is similar to, but different from, the Building Block method of instruction (based on Skinner's idea of shaping) currently taught to flight instructors (Aviation Instructor's Handbook, 1977). Both create an environment that reduces failures and allows success. In shaping, the final task or behavior is broken down into a series of simplified approximations to the final behavior, and each one is successfully learned before moving on to the next approximation. In scaffolding, the task or behavior is not simplified or broken 
down into approximations. Rather, the scaffolding holds the task constant and simplifies the learner's role through graduated intervention of the teacher (Greenfield, 1984).

Again, this fits the case with SA. For example, as a beginning flight student learns to keep track of the airplane's location, the instructor has a greater responsibility initially because the student is weak in this area.

\section{Cognitive Apprenticeship}

Bringing together the concepts of indexicality of knowledge, scaffolding, and proleptic instruction, Brown et al. (1989) coined the term "cognitive apprenticeship." The term apprenticeship is used to emphasize the importance of authentic activity in learning and knowledge and to highlight the "inherently context-dependent, situated, and enculturating nature of learning." ( $p$ 39). The term cognitive implies that apprenticeship techniques can be applied beyond the physical skills usually associated with apprenticeship to cognitive skills.

\section{Learning Situation Awareness}

Based on the educational theory of situated cognition, Young (1993) has identified four tasks for designing situated learning: (a) selecting the situations, (b) providing scaffolding for the student, (c) determining and supporting the role of the teacher, and (d) assessing situated learning. These four tasks, combined with Stokes' theory of schema matching and the general philosophy of situated learning, give five ways in which situated learning can be applied to help flight students learn SA.

First, the context-dependent nature of knowledge and learning must be recognized. SA requires developing a repertoire of schemata developed through interacting with the an authentic environment. Flight training needs to provide the opportunity to develop as many of these schema as possible. The idea that SA can only be gained through experience is correct, but many of the experiences can be provided during flight training.

Second, both simulator and flight lessons must be made as authentic as possible by anchoring them to realistic flight scenarios. Selecting the situations is important so that the curriculum sets up opportunities for events to unfold and students can engage in practice. The schemata that the student develops in training can then be built around realistic occurrences.

Third, a learning environment must be designed to include the richness of the authentic environment instead of trying to separate the salient features from the noise. Students should be given the opportunity to observe expert pilots who are operating in the environment. Through proleptic instruction, students learn from experts which cues are relevant in different circumstances. 
Fourth, the lessons must be designed to keep the SA portion constant and have the instructor provide the scaffolding needed so the student can perform successfully in the authentic environment. Instructors should be provided with training so they understand and use the principle of scaffolding. The students should be working just beyond what they are able to do alone and gradually handed off responsibility for maintaining SA.

Finally, assessment techniques should be defined that measure the student's SA skills.

\section{Summary}

Situation Awareness is recognized as a cognitive skill important to the safety of aviation. However, currently no known curricula specifically include SA training in the flight curriculum of novice pilots. The educational theory of situated learning fits well with the task of SA and could be used as a foundation for developing a method to help students learn SA. Based on situated learning, designing flight training that includes learning SA should be based on authentic situations in which students can develop schemata that capture the interrelationship of events. Students should be working at a level beyond what they can do alone by collaborating with the instructor. Instructional techniques such as scaffolding should be used to provide support to students, as needed, with a gradual reduction of that support as the student takes over responsibility. Finally, the flight training should be developed so that students, being novices, can learn a great deal from observing their instructor. This can be exploited in the area of SA. Future research should be done to evaluate the effectiveness of a curriculum that attempts to improve SA of novice pilots through flight lessons based on situated learning theories.

\section{References}

Aviation Instructor's Handbook (AC 60-14). (1977). U.S. Dept. of Transportation, Federal Aviation Administration.

Brown, J. S., Collins, A., \& Duguid, P. (1989). Situated cognition and the culture of learning. Educational Researcher, 18(1), 32-42.

Brown, J. S., \& Duguid, P. (1993). Stolen knowledge. Educational Technology, 33(3), $10-$ 15.

Crabtree, M. S., Marcelo, R. A. Q., \& McCoy, A. L. (1993). An examination of a subjective situational awareness measure during training on a tactical operations simulator. Proceedings of the Seventh International Symposium on Aviation Psychology (pp. 891895).

Edens, E. S. (1991). Individual differences underlying pilot cockpit error (Doctoral dissertation, George Mason University, 1991). Dissertation Abstracts International. $52 / 10-B, 5567$.

Endsley, M. R. (1988). Design and evaluation for situation awareness enhancement. Proceedings of the Human Factors Society 32nd Annual Meeting (pp. 97-101).

Endsley, M. R. (1990a). Predictive utility of an objective measure of situation awareness. Proceedings of the Human Factors Society 34th Annual Meeting (pp. 41-45). 
Endsley, M. R. (1990b). Situation awareness in dynamic human decision-making: theory and measurement. Dissertation Abstracts International, 52/01-B p 555.

Endsley, M. R. (1993). Situation awareness and workload: flip sides of the same coin. Proceedings of the Seventh International Symposium on Aviation Psychology. (pp. 906-911).

Forsyth, D. L. \& Shaughnessy, J. D. (1978). Single Pilot IFR Operating Problems Determined from Accident Data Analysis (NASA Technical Memorandum 78773). Hampton, VA: Langley Research Center.

Fracker, M. L. (1988). A theory of situation assessment: implications for measuring situation awareness. Proceedings of the Human Factors Society 32nd Annual Meeting (pp. 102-106).

Greenfield, P. M. (1984). A theory of the teacher in the learning activities of everyday life. In B. Rogoff \& J. Lave (Eds.), Everyday cognition: Its development in social context (pp. 117-138). Cambridge, MA: Harvard University Press.

Hartman, O. H., \& Secrist, G. E. (November, 1991). Situation awareness is more than exceptional vision. Aviation, Space, and Environmental Medicine, pp. 1084-1089.

Instrument Commercial Manual (8th ed.). (1994). Englewood, CO: Jeppesen Sanderson.

Kass, S. J., Herschler, D. A., \& Companion, M. A. (1991). Training situation awareness through pattern recognition in a battlefield environment. Military Psychology, 3(2), $105-112$.

Kershner, W. (1989). The Flight Instructor's Manual (2nd ed.). Ames, IA:Iowa State University Press.

Lave, J., \& Wenger, E. (1991). Situated Learning: Legitimate Peripheral Participation. Cambridge: Cambridge University Press.

Rogoff, B., \& Gardner, W. (1984). Adult Guidance of Cognitive Development. In B. Rogoff \& J. Lave (Eds.), Everyday cognition: Its development in social context (pp. 93-116). Cambridge, MA: Harvard University Press.

Schwartz, D. (1987). Training for Situational Awareness. Paper presented to The Flight Safety Foundation 40th International Air Safety Seminar, Tokyo, Japan.

Stokes, A. F., Kemper, K. L., \& Marsh, R. (1992). Time-stressed flight decision making: $a$ study of expert and novice aviators. (Tech. Rep. No. ARL-93-1/INEL-93-1). Savoy, IL: University of Illinois at Urbana-Champaign, Aviation Research Laboratory, Institute of Aviation.

Young, M. F. (1993). Instructional design for situated learning. Educational Technology, Research, and Development, 41(1), 43-58. 